http://jmscr.igmpublication.org/home/ ISSN (e)-2347-176x ISSN (p) 2455-0450

crossref DOI: https://dx.doi.org/10.18535/jmscr/v8i12.13

\title{
The Study of Clinical Profile of Non-Traumatic Paraplegia in a Tertiary Care Hospital in North Andhra
}

\author{
Authors \\ Chintada Sruthikeerthi', K Manoj Kumar², YGS Raju ${ }^{3}$ \\ ${ }^{1}$ Post graduate, Department of General medicine, Andhra medical college, Visakhapatnam \\ ${ }^{2}$ Assistant Professor, Department of General Medicine, Andhra Medical College, Visakhapatnam \\ ${ }^{3}$ Professor, Department of General Medicine, Andhra Medical College, Visakhapatnam
}

\begin{abstract}
Background: Paraplegia can occur due to traumatic or non-traumatic pathology. Myelopathy a broad term that refers to spinal cord involvement of multiple etiologies. Spinal cord diseases often have devastating consequences due to its confinement in a very small area. Many of these diseases are potentially reversible if they are recognized on time, hence the importance of recognizing the significance of magnetic resonance imaging when approaching a multifactorial disease considered as one of the most critical neurological emergencies, where prognosis depends on an early and accurate diagnosis. This study aimed to identify the clinical profile of non-traumatic paraplegia in tertiary care hospital.

Material and Methods: 30 patients of non-traumatic paraplegia were studied at King George hospital, Visakhapatnam from March 2019 to April 2020. Patients underwent a detailed clinical evaluation followed by laboratory investigations and neuroimaging studies.

Results: Present study showed male preponderance with $M: F=1.7: 1$. Incidence was common in productive age groups of $3 \mathrm{rd}$ and 4th decade. Presenting symptoms included weakness of lower limbs (100\%), sensory symptoms (52\%), retention of urine (57\%), backache (23\%), incontinence of urine $(23 \%)$ and fever (17\%). Tuberculosis of spine was the commonest cause of paraplegia followed by Transverse myelitis; Disc prolapse.

Conclusion: Tuberculosis was the commonest cause of non-traumatic paraplegia. Transverse myelitis of probable viral etiology was the second commonest cause.

Keywords: Non traumatic paraplegia; transverse myelitis; Potts spine.
\end{abstract}

\section{Introduction}

Paraplegia can occur due to traumatic or nontraumatic pathology. The term myelopathy describes pathologic conditions that cause spinal cord, meningeal or peri meningeal space damage or dysfunction. Traumatic injuries, vascular diseases, infections and inflammatory or autoimmune processes may affect the spinal cord $^{(1)}$ due to its confinement in a very small space. Spinal cord injuries usually have devastating consequences such as quadriplegia, paraplegia and severe sensory deficits. The history, an adequate neurological examination and the study of the cerebrospinal fluid (CSF) guide the diagnosis of spinal cord injuries. However, imaging is of great importance in the diagnosis and to classify the etiology appropriately ${ }^{(2-3)}$. Many of the processes affecting the spinal cord 
may be reversible if recognized and treated early. The vast majority of spinal cord diseases may be treated medically, with surgical treatment reserved for compressive disorders, which constitute a neurological emergency ${ }^{(4)}$.

\section{Aims and Objectives}

1) To determine the etiological factors prevalent in and around Visakhapatnam contributing to non- traumatic paraplegia.

2) The etiological subcategorization of various non-traumatic paraplegia.

3) To study the clinical pattern of evolution and presentation of nontraumatic paraplegia.

\section{Methodology \\ Inclusion Criteria}

1) Age more than 12 years .

2) Acute and subacute to chronic onset motor weakness of lower limbs of non-traumatic aetiology.

\section{Exclusion Criteria}

1) Age less than 12 years.

2) Patients having traumatic paraplegia.

3) Patients who presented with history of weakness of lower limbs but on examination if the upper limbs were also involved than those patients were excluded.

Each case was subjected to the following investigations. Hemogram with peripheral smear, Blood sugar and urea, Urine for routine microscopic and chest radiograph (PA), Tuberculin test, ESR, Sputum AFB, Serum albumin, lymph node biopsy, liver function test, sonography of abdomen and pelvis, mammography, ECG, NCV, HIV etc. X-ray spine: In various views at different levels, based on clinical localization of lesion. MRI spine, MRI brain when required. Lumbar puncture for CSF examination for routine micro, routine sugar, AFB, gram stain, VDRL, culture and sensitivity. Methods used for statistical analysis: Statistical analysis was done using EPI Info Version-6.
Percentage, Chi-square, 'p' value were calculated wherever applicable.

\section{Results}

A total of 30 cases were studied during March 2019 to April 2020 at King George hospital, Visakhapatnam. There was male preponderance, male: female - 1.7: 1. Majority of cases belongs to productive age groups of 3rd and 4th decade. $46.6 \%$ had acute presentation while $53.3 \%$ had subacute to chronic onset. Progression were gradual in $66.6 \%$ while static in $33.3 \%$. Clinical features among patients were shown in table 1 and etiologies in table 2. Hypertonia with hyperreflexia noted in $43 \%$ while $57 \%$ had hypotonia with hyporeflexia. All modalities of sensation were lost with definitive upper level in $43.3 \%$ while sensory loss patch in $10 \%$, impaired in $36 \%$ and no sensory disturbances in $11 \%$. Spinal examination revealed tenderness in $33 \%$ and gibbus in $13.3 \%$ of patients.

Table 1: Frequency distribution of various clinical features

\begin{tabular}{|l|c|}
\hline Clinical feature & Percentage \\
\hline Weakness of lower limbs & $100 \%$ \\
\hline Sensory symptoms & $52 \%$ \\
\hline Retention of urine & $57 \%$ \\
\hline Incontinence of urine & $23 \%$ \\
\hline Low backache & $23 \%$ \\
\hline Constipation & $22 \%$ \\
\hline Fever & $17 \%$ \\
\hline Spine deformities & $13.3 \%$ \\
\hline Constitutional symptoms & $16 \%$ \\
\hline
\end{tabular}

Table 2: Frequency distribution of etiologies among patients

\begin{tabular}{|l|c|c|}
\hline Etiology & $\begin{array}{c}\text { Number of } \\
\text { Cases }\end{array}$ & Percentage \\
\hline Potts Spine & 11 & $36.6 \%$ \\
\hline Acutetransversemyelitis & 9 & $30 \%$ \\
\hline PID Combined & 3 & $10 \%$ \\
\hline Secondaries Sinus & 2 & $6.6 \%$ \\
\hline $\begin{array}{l}\text { Subacute Saggital } \\
\text { Degeneration }\end{array}$ & 1 & $6.67 \%$ \\
\hline Toxins: Organophosphorous & 1 & $3.3 \%$ \\
\hline Leriche's Syndrome & & $3.3 \%$ \\
\hline $\begin{array}{l}\text { Superior Shrombosis } \\
\text { Throm }\end{array}$ & \\
\hline
\end{tabular}

In CSF protein was raised in $56 \%$ of cases, sugar was normal in all cases and elevated Lymphocytes were seen in $28 \%$ cases. MRI spine showed 
secondaries in vertebrae in patients showing wedging with osteolysis on $\mathrm{X}$ ray spine. In our study, Tuberculosis was the commonest $(36.6 \%)$ cause of non-traumatic paraplegia which includes Pott's spine, paravertebral abscess, arachnoiditis, transverse myelitis and granuloma. Second most common was Transverse myelitis of nontuberculous origin which include HIV, HSV 1, HSV 2 infections, Autoimmune disorder(SLE), demyelination disorder Neuromyelitis Optica spectrum disorder (NMOSD). Prolapsed Intervertebral Disc (PID) (10\%) is the third most common cause of paraplegia. Secondaries in vertebrae, Subacute Combined Degeneration of spinal cord, superior sagittal sinus thrombosis, leriche's syndrome, organophosphorus poisoning are less frequent causes of nontraumatic paraplegia. Cord compression was suspected in most patients clinically and had similar lesion found on MRI. Out of which 1 case showed demyelinating lesion \& two cases showed normal MRI. Most common cause of cord compression on MRI was Pott's spine, metastasis of vertebrae \& PID. MRI is most useful test in diagnosis of cause of paraplegia. Out of 30 patients, 4 patients (6\%) expired, of whom 2 had TBM with hydrocephalus, whereas 1 was known case of breast cancer with multiple metastasis and one patient with aortoiliac occlusive disease (Leriche's). The other 26patients were discharged from Medicine ward and on follow upand some are transferred to Orthopaedics or neurosurgery department for surgical intervention.

\section{Discussion}

We enrolled 30 patients with non-traumatic paraplegia during period from March 2019 to April 2020. Out of 30 males to female ratio was 1.7:1, which was similar comparable to other studies. Comparison of mode of onset was shown in table 3 among various studies.
Table 3: Comparison of mode of onset among various studies

\begin{tabular}{|l|c|c|c|}
\hline Mode of onset & $\begin{array}{c}\text { Choudary et al } \\
(6)\end{array}$ & $\begin{array}{c}\text { Bharghav and } \\
\text { Berry (7) }\end{array}$ & $\begin{array}{c}\text { Present } \\
\text { study }\end{array}$ \\
\hline Acute & $17.9 \%$ & $26 \%$ & $46.6 \%$ \\
\hline $\begin{array}{l}\text { Subacute to } \\
\text { chronic }\end{array}$ & $70.6 \%$ & $74 \%$ & $53.3 \%$ \\
\hline
\end{tabular}

In present study all the patients had weakness of lower limbs, bladder disturbances were observed in $80 \%$ followed by backache (24\%), fever (18\%) and tingling and numbness (4\%). Choudhary et $\mathrm{al}^{(6)}$ reported to have observed backache in $26 \%$, bladder disturbance in $43 \%$ and paraesthesia in $21 \%$ of patients. In the present study $80 \%$ of the patient had bladder disturbance and $20 \%$ without any bladder disturbance, among these $57 \%$ had retention and $23 \%$ had incontinence. At presentation flaccid features predominated with $57 \%$ of patients having hypotonia and hyporeflexia, $43 \%$ had hypertonia and hyperreflexia. Spinal examination revealed $58 \%$ to be normal, tenderness was present in 33\%, Gibbus was present in $13.3 \%$ and scoliosis was seen in one patient (2\%). CSF analysis could be done only in 10 cases. In patients whom can be diagnosed radiologically like Pott's spine, PID, secondaries, CSVT, Leriche's syndrome CSF analysis was not done. CSF protein was raised in $56 \%$ of cases. There is no association between CSF sugar and etiology of paraplegia. With imaging study, Plain radiograph of the spine was undertaken in 14 cases. In $42.85 \%$ of these cases it was normal. In $36.6 \%$ of the cases of tuberculosis destruction of the vertebral bodies, compression and intervertebral disc space narrowing was seen. Plain x-rays of spine were contributory to the diagnosis in $56 \%$ of cases in present series. The plain $\mathrm{x}$ - rays were found to be diagnostic or contributory in $53 \%$ of cases by Ghosh et al and $44 \%$ by Rao and Dinakar et $\mathrm{al}^{(8)}$. MRI spine was subjected in all patients. One patient had longitudinal extension transverse myelitis involving more than 3 vertebrae, 21 had myelitis and was normal in 2 patients $(6.6 \%)$, Anterior spinal artery thrombosis in 1 case (3.33\%), inflammatory spondylodiscitis with paraspinal 
abscess in 2 cases $(6.66 \%)$. In one patient MR venogram brain suggestive of superior sagittal sinus thrombosis.

\section{Conclusion}

1) In hospital incidence, Non-traumatic paraplegia has aninpatient incidence of 0.7 cases/1000 during the study period and has a significant burden on health resources.

2) In this study of Non traumatic paraplegia the incidence was more in Males and was more common in the productive age groups of 3rd and 4th decade.

3) Tuberculosis was the commonest cause.

4) Transverse myelitis of probable viraletiology was the second commonest cause.

5) Other causes like neoplastic, Disc prolapse, superior sagittal sinus thrombosis, aortoiliac occlusive disease were also seen but to a lesser extent.

\section{Limitations}

The study is done in a limited number of patients in one region. Results may vary in a large number of subjects and various regions of people.

\section{References}

1. Myelopathy. Diseases Database Ver 1.8. Medical lists and links [internet]. 2006 [citado: 2 de julio del 2011]. Disponible en:

http://www.diseasesdatabase.com/umlsdef. asp?glngUserChoice $=22984$.

2. Hauser SL. Diseases of the spinal cord. En: Harrison's principles of internal medicine. 16th ed. New York: McGrawHill; 2005. p. 2438-47.

3. García DR. Mielopatías. Manual de Prácticas Médicas-Hospital Hermanos Ameijeiras [internet]. 2008 [citado: 18 de junio del 2011].
4. Scotti G, Gerevini S. Diagnosis and differential diagnosis of acute transverse myelopathy. The role of neuroradiological investigations and review of the literature. Neurol Sci. 2001;22Suppl 2: S69-73.

5. Venkatraman S, Dhamija RM, Sanchetee PC et al: Progress in clinical neurosciences 1990; 6(2) :275- 297.

6. Chowdhary KN.et al. Paraplegia-clinical and etiological study. J. Ass. Phy. Ind. 1998; $16: 751$.

7. Bhargava, Berry. Paraplegia. J. Ass. Phy.India. 1961; 9:211.

8. Rao PB, Dinakar L. Spinal compression. J. Ass. Phy. India 1970; 18:1009. 